\title{
An Investigation of a Palladium Catalysed Biaryl Synthesis of Pyrrolophenanthridine Derivatives. Extension of the Heck Reaction.
}

\author{
Simon J. Garden*, José C. Torres and Angelo C. Pinto \\ Instituto de Química, Departamento de Química Orgânica, Bloco A, \\ Universidade Federal do Rio de Janeiro, 21945-970, Rio de Janeiro - RJ, Brazil.
}

\begin{abstract}
Derivados halogenados de $N$-benzilisatinas ciclizaram facilmente, com eliminação de haletos de hidrogênio, numa reação de síntese de biarilas sob catálise por paládio, na ausência de ligantes fosfinas, levando às oxopirrolo[3,2,1-de]fenantridinas correspondentes.
\end{abstract}

$N$-Benzylisatin derivatives have been found to readily undergo cyclisation via hydrogen halide elimination in a biaryl synthesis reaction when using palladium catalysts, in the absence of phosphine ligands, to yield oxopyrrolo[3,2,1-de]phenanthridine derivatives.

Keywords: Heck reaction (Jeffery's conditions), benzylisatins, pyrrolophenanthridines, biaryl coupling

\section{Introduction}

The pyrrolo[3,2,1-de]phenanthridine skeleton has been found in a large number of natural products from the plants of almost all of the genera of the family Amaryllidaceae. Examples of these compounds include assoanine ${ }^{1}(\mathbf{1})$, oxoassoanine $^{2}(\mathbf{2})$, hippadine $^{3}(\mathbf{3})$ and ungeremine ${ }^{4}(\mathbf{4})$ amongst others ${ }^{5}$. These compounds (Figure 1 ) are of interest as synthetic targets not only for the development of synthetic methodology but also for their biological activities ${ }^{6}$. An important feature of the pyrrolo[3,2,1-de]phenanthridine skeleton is the biaryl linkage and this has often been a central subject of the synthetic methodology employed for the construction of these, and a diverse range of phenanthridine or benzophenanthridine derivatives ${ }^{7}$.

The Heck reaction ${ }^{8}$ is a versatile methodology for the formation of new C-C bonds, particularly for the coupling of an aromatic halide with an olefin ${ }^{9}$. The corresponding biaryl synthesis where hydrogen halide is eliminated from the two aromatic components is a recent extension of the Heck reaction ${ }^{10}$, whereas the corresponding biaryl synthesis using an oxidative addition reaction with stoichiometric quantities of palladium salts has been more extensively studied ${ }^{11}$.

\section{Results and Discussion}

In a general approach for the construction of pyrrolo-

*e-mail: garden@iq.ufrj.br
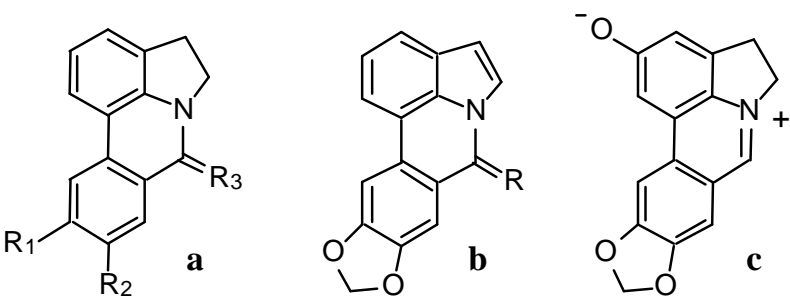

Figure 1. Structures of some naturally occurring pyrrolophenanthridine derivatives. a) $\mathrm{R}_{1}=\mathrm{R}_{2}=\mathrm{OCH}_{3}, \mathrm{R}_{3}=2 \mathrm{H}$, Assoanine (1); $\mathrm{R}_{1}$ and $\mathrm{R}_{2}$ as for $\mathbf{1}, \mathrm{R}_{3}=\mathrm{O}$, Oxoassoanine (2); $\mathrm{R}_{1} / \mathrm{R}_{2}=\mathrm{CH}_{2}(\mathrm{O})_{2}, \mathrm{R}_{3}=\mathrm{O}$, Anhydrolycorine-7-one (7); b) $\mathrm{R}=\mathrm{O}$, Hippadine (3); $\mathrm{R}=2 \mathrm{H}$, Dehydroanhydrolycorine (6); c) Ungeremine (4).

phenanthridine alkaloids an appropriately substituted $\mathrm{N}$-benzylisatin derivative (5), that contained the entire carbon framework, was envisaged as a suitable precursor for the obtention of these natural products and their analogs (Scheme 1).

Subsequent reduction of the cyclised isatin derivatives to indoles would yield, for example, dehydroanhydrolycorine ${ }^{12}$ (6). Successful cyclization would also furnish substrates to further on going investigations of the use of $\mathrm{BH}_{3}$.THF for the reduction of isatin derivatives to indoles ${ }^{13}$. Strategically, oxidation of the derivatives of compound $\mathbf{6}$ could present problems due to oxidation of the indolic double bond. However, Gribble reduction ${ }^{14}$ of the indolic double bond and subsequent oxidation of the indoline would seem to be a viable route for the synthesis of, for example, anhydrolycorin7-one (7) and hippadine (3), Scheme 1. 

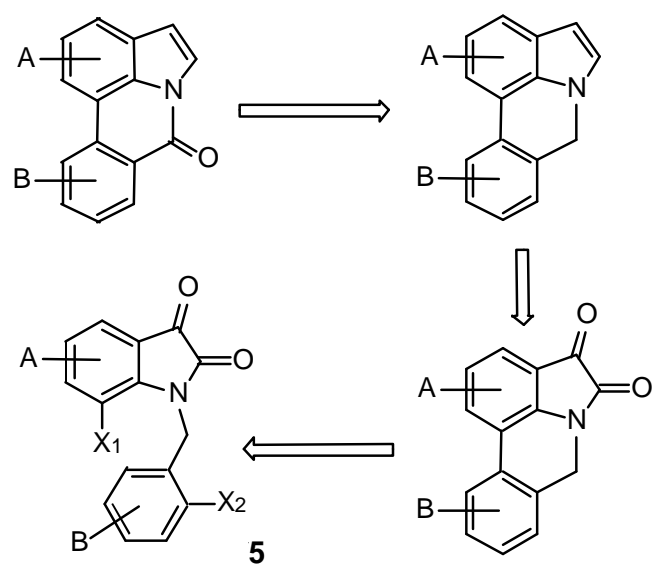

Scheme 1. Retrosynthetic analysis for the synthesis of pyrrolophenanthridine derivatives.

The appropriate $\mathrm{N}$-benzylisatin derivatives 5 for investigation of the synthesis of the pyrrolphenanthridines were readily prepared by benzylation of substituted isatin derivatives ${ }^{15} \mathbf{8}$ using basic conditions (Scheme 2).

Initial experiments to determine conditions for the formation of the biaryl linkage focused upon the attempted cyclisations of 7-bromo- and 7-iodo-5-methyl- $N$ benzylisatin (5a and $\mathbf{5 b}$ ) using $10 \mathrm{~mol} \% \mathrm{Pd}(\mathrm{OAc})_{2}$ in the presence of $20 \mathrm{~mol} \%$ of triphenylphosphine in acetonitrile ${ }^{16}$. Substantial substrate consumption was observed but no defined product could be isolated upon workup. It was therefore questioned as to whether the cis dicarbonyl system could possibly interfere in the coupling reaction. An inspection of the isatin literature revealed a study by Martinez and Naarmann that reported the Suzuki couplings of a number of heteroarylboronates with 5-bromo- and 5iodo- isatin using tetrakis-triphenylphosphine palladium as the catalyst, albeit in very modest yields ${ }^{17}$. A plausible explanation for the modest yields obtained by Martinez and Naarmann and the poor mass balance in our reactions, where the substrate was recovered in $52-62 \%$ yield, was encountered in the work of Lathourakis and Litinas ${ }^{18}$, where these authors have shown that $\mathrm{PPh}_{3}$ reacts with isatin to yield a phosphoranylidene that can undergo Wittig reactions. Therefore, to eliminate this source of interference the ketone was transformed into the corresponding ketal (9a-i) using conventional methodology (Scheme 2).

Submission of $\mathbf{9}$ (a and $\mathbf{b})$ or $N$-benzyl-5-methyl-7-iodo2-oxindole, obtained by reduction of $\mathbf{5 b}$ with hydrazine hydrate $^{19}$, to the same reaction conditions ${ }^{16}$ as used in the first experiments also yielded no defined products but the substrates were recovered in $87-91 \%$ yield. The reaction conditions $^{16}$ were subsequently modified by replacing acetonitrile with DMF to allow an increased reaction temperature. When $\mathbf{9 b}$ was submitted to the new

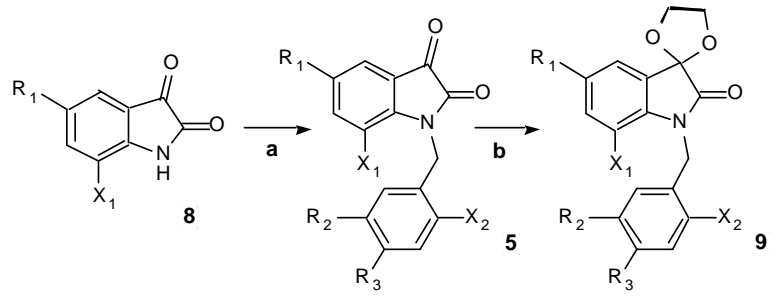

Scheme 2. Synthesis of cyclization substrates. a) $\mathrm{CaH}_{2}$ or $\mathrm{K}_{2} \mathrm{CO}_{3}$, benzyl halide, DMF; b) $\left(\mathrm{CH}_{2} \mathrm{OH}\right)_{2}$, PTSA, toluene; for definition of the substituents $\left(R_{1}\right.$ to $R_{3}$ ) see Table 1 , if $X_{1}$ is a halogen (I or Br) then $\mathrm{X}_{2}$ is a hydrogen (or vice versa).

conditions, the formation of defined products could be observed upon TLC analysis. ${ }^{1} \mathrm{H}$ NMR analysis of the crude product after workup of the reaction revealed the conversion of the reaction to be low, $29 \%$ ( $96 \%$ mass balance), and the major product was identified as the reduced $N$-benzyl5 -methylisatin ethylene ketal (25\%) from comparison of spectral data with an authentic sample ${ }^{20}$. The structure of the minor component (4\%) was suspected to be the desired cyclised product 10a; this suspicion being latter confirmed, vide infra. Subsequent investigations of the reaction conditions were based upon experimental work first reported by Jeffery ${ }^{21}$. Similarly to the initial experiments using the substrates 5a and 5b under Heck conditions, substrate consumption was observed but no defined products could be detected by TLC or upon workup of the reaction. Attention was then focused upon the ketal substrate 9a, which in the presence of $10 \% \mathrm{Pd}(\mathrm{OAc})_{2}\left(0.1 \mathrm{~mol} \mathrm{~L}^{-1} \mathrm{DMF}\right), 1$ mole equivalent of $\mathrm{Bu}_{4} \mathrm{NBr}$, and 5.5 mole equivalents of KOAc at $100^{\circ} \mathrm{C}$ (bath temp.) resulted in the formation of a single product at 56\% conversion of the substrate, Table 1 , entry 1 (95\% mass balance). This product had the same NMR features as the minor product observed in the previous experiment using this substrate, vide supra. In a similar experiment where $\mathrm{PdCl}_{2}$ was used as the catalyst (table entry 2) a comparable result was obtained with a $98 \%$ mass balance. In contrast to the bromide $9 \mathbf{a}$, the iodide $9 \mathbf{b}$ readily furnished the same product as the previous experiments. Isolation and characterisation of this product identified it as the oxopyrrolophenanthridine 10a. Investigation of the catalyst loading revealed a reaction time dependance upon the reaction (table entries 4-7). The ortho-iodobenzyl derivative $9 \mathbf{c}$ cyclised under similar conditions to yield the parent ring system 10b (table entry 8). Further evidence for halogen positional versatility can be seen in table entries 9 and 10 where the oxopyrrolophenanthridine derivative 10c was obtained in 77-78\% yield from either substrate $9 \mathbf{d}$ or $9 \mathbf{e}$. The iodides were found to be superior to the bromides giving better yields and shorter reaction times (compare table entries 1 and 2 with 5, 10 with 11, and 12 with 13). The presence of a nitro group either on the halogenated aromatic 
ring or the aromatic ring to be attacked by the intermediate organopalladium species significantly accelerated the reaction giving increased yields in reduced reaction times (compare table entries 1, 2 and 3 with 9 and 10). Little difference in reaction time or yield was observed in the cyclisations of the unsubstituted $9 \mathbf{c}$ and the methylenedioxy substituted 9h (table entries 8 and 13). However, the dimethoxy substituted 9i required a signifcantly longer reaction time to attain an almost equally good yield (table entry 14).
In all successful reactions initiation of the reaction was evidenced by a darkening of the reaction mixture which normally had an onset temperature greater than $80^{\circ} \mathrm{C}$ (bath temp.). Both aromatic iodides and bromides have been cyclised successfully in the absence of phosphine ligands, though the experiments revealed that the iodides are better substrates ${ }^{22}$. No special precautions are required for performing these cyclisations which have been routinely performed exposed to the laboratory atmosphere.

Table 1. Palladium catalysed cyclization of N-benzylisatin derivatives

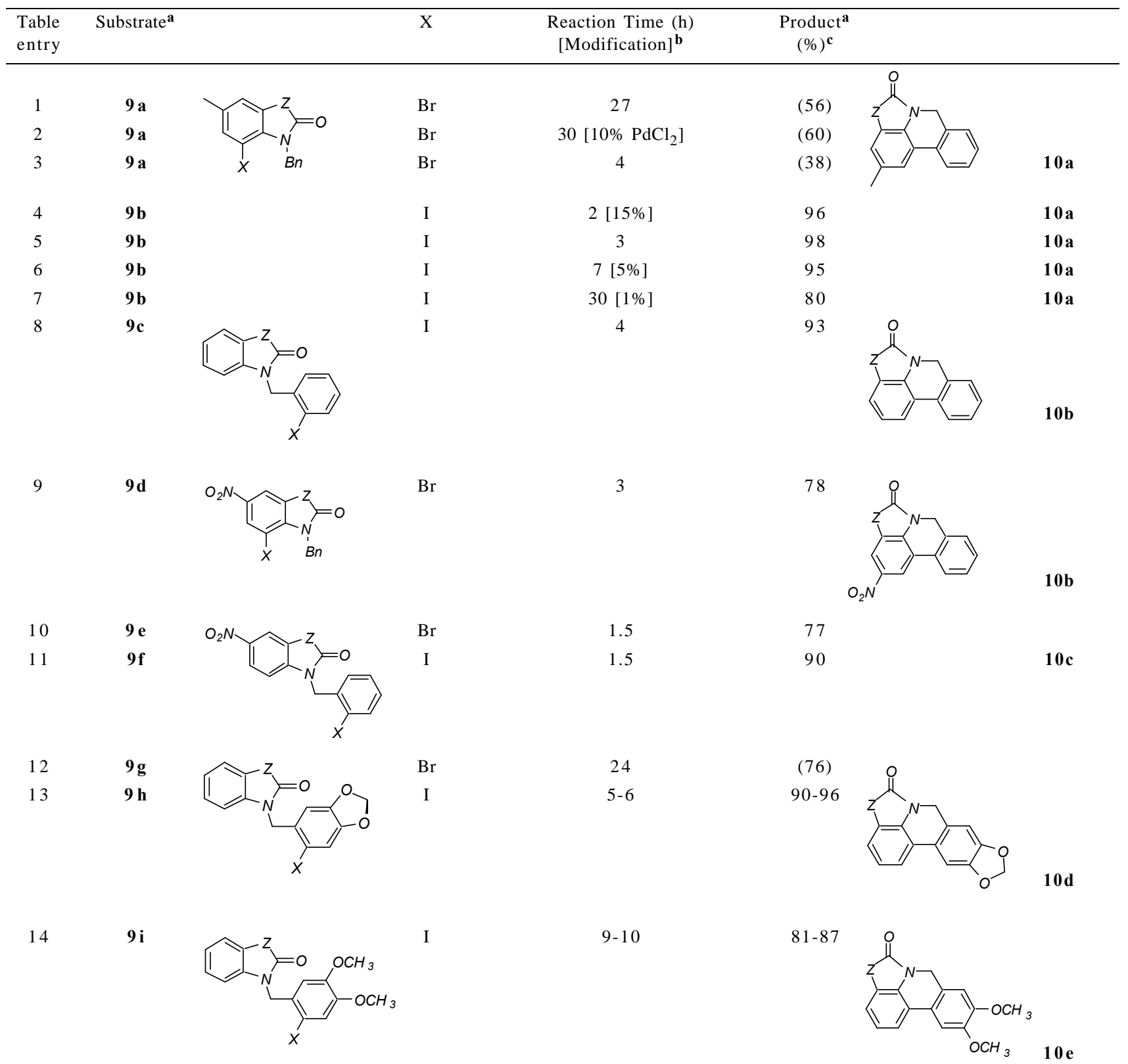

a $\mathrm{Z}=$ spiro-2-(1,3-dioxolane) ring; b Reaction conditions used: 10 mole\% $\mathrm{Pd}(\mathrm{OAc})_{2}, 1.1$ equiv. $\mathrm{Bu}_{4} \mathrm{NBr}, 5.5$ equiv. KOAc, $\mathrm{DMF}$ (substrate concentration $\left.0.1 \mathrm{~mol} \mathrm{~L}^{-1}\right), 100^{\circ} \mathrm{C}$. Modifications to this procedure are indicated within the square brackets, [modification]. All reactions were accompanied by silica gel TLC until substrate was no longer evident or until the reaction appeared to no longer progress; c Isolated yield of purified product. Values in parenthesis are conversions to cyclised product based upon isolation of the reaction product(s) and substrate as calculated from analysis of ${ }^{1} \mathrm{H}$ NMR spectra. 
The reaction offers a unique opportunity to investigate structural requirements and substituent effects as it has been found to be tolerant to both activation and deactivation of the aromatic nuclei and therefore, mechanistically relevant information can be delineated. These studies as well as the application of this methodology to the synthesis of a number of natural products are currently being investigated.

\section{Experimental}

\section{General}

${ }^{1} \mathrm{H}$ and ${ }^{13} \mathrm{C}$ NMR spectra were recorded using $300 \mathrm{MHz}$ and $200 \mathrm{MHz}\left({ }^{1} \mathrm{H}\right)$ Bruker spectrometers and are referenced to TMS. Coupling constants are quoted in Hz. Mass spectra were recorded at NPPN (Nucleo de Pesquisa de Produtos Naturais, Universidade Federal do Rio de Janeiro) using a Hewlett-Packard GC-MS or by direct insertion using a VG Autospec. Infra-red spectra were recorded using a Perkin Elmer 1600 FT-IR (error $\pm 4 \mathrm{~cm}^{-1}$ ) as $\mathrm{KBr}$ discs. Melting points were recorded on a hotstage microscope and/or using a MelTemp II capillary apparatus and are reported as uncorrected values. DMF was used as supplied without further treatment.

\section{Experimental procedure}

The appropriate isatin derivative $(1.00 \mathrm{mmol}), \mathrm{Pd}(\mathrm{OAc})_{2}$ $(0.10 \mathrm{mmol}), \mathrm{Bu}_{4} \mathrm{NBr}(1.10 \mathrm{mmol}), \mathrm{KOAc}(5.50 \mathrm{mmol})$ and DMF $(10 \mathrm{~mL})$ were added to a round bottom flask. The reaction mixture was heated on an oil bath $\left(100 \pm 5^{\circ} \mathrm{C}\right.$, bath temp.) and the reaction accompanied by TLC. On complete reaction, distilled water $(50 \mathrm{ml})$ or aqueous $\mathrm{HCl}\left(0.2 \mathrm{~mol} \mathrm{~L}^{-1}\right.$, $50 \mathrm{~mL}$ ) was added to the reaction mixture and the mixture was extracted with ethyl acetate $(3 \times 15 \mathrm{~mL})$. The organic phase was dried over $\mathrm{Na}_{2} \mathrm{SO}_{4}$, filtered and concentrated under reduced pressure. The residue was then chromatographed on a silica gel column using hexane/ethyl acetate $(4: 1, \mathrm{~V} / \mathrm{V})$ as eluent. Physical and spectroscopic data of compounds 10 follow:

spiro-4-(ethylenedioxy)-2-methyl-7-hydropyrrolo [3,2,1-de]phenanthridin-5-one (10 a)

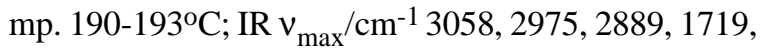
1636, 1449, 1349, 1204, 1029, 1000, 948, 864, 773, 741 (KBr), Mass [m/z (abun.)] 293(33\%), 264(100), 220(29), 193(27), 178(4), 165(17), 110(31), 96(29), 96(29), 82(28); ${ }^{1} \mathrm{H} \mathrm{NMR}\left(300 \mathrm{MHz}, \mathrm{CDCl}_{3}\right) \delta 2.34\left(\mathrm{~s}, 3 \mathrm{H}, \mathrm{CH}_{3}\right), 4.29-4.34$ (m, $2 \mathrm{H}, \mathrm{CH}_{2}$ ), 4.56-4.61 (m, 2H, $\left.\mathrm{CH}_{2}\right), 4.88\left(\mathrm{~s}, 2 \mathrm{H}, \mathrm{CH}_{2}\right.$ ), $7.06(\mathrm{~d}, 1 \mathrm{H}, \mathrm{CH}, J 0.6 \mathrm{~Hz}), 7.10$ (dd, $1 \mathrm{H}, \mathrm{CH}, J 0.9,6.3 \mathrm{~Hz}$ ),
7.24 (dt, 1H, CH, J 1.4, 7.4, 7.4 Hz), 7.26 (dt, 1H, CH, J 0.7, $7.4,7.4 \mathrm{~Hz}), 7.43(\mathrm{~s}, 1 \mathrm{H}, \mathrm{CH}), 7.67(\mathrm{~d}, 1 \mathrm{H}, \mathrm{CH}, J 7.5 \mathrm{~Hz}) ;{ }^{13} \mathrm{C}$ NMR PENDANT ( $\left.75 \mathrm{MHz}, \mathrm{CDCl}_{3}\right) \delta 21.6\left(\mathrm{CH}_{3}\right), 44.5\left(\mathrm{CH}_{2}\right)$, $66.0\left(\mathrm{CH}_{2}\right), 103.4(\mathrm{C}), 117.2(\mathrm{C}), 122.4(\mathrm{CH}), 122.7(\mathrm{C}), 124.6$ $(\mathrm{CH}), 125.1(\mathrm{CH}), 127.8(\mathrm{CH}), 128.1(\mathrm{CH}), 128.2(\mathrm{C}), 128.5$ $(\mathrm{CH}), 128.9(\mathrm{C}), 133.3(\mathrm{C}), 137.1(\mathrm{C}), 172.9(\mathrm{C}=\mathrm{O})$. Found: C, 73.23; $\mathrm{H}, 5.04 ; \mathrm{N}, 4.62$. Calc. for $\mathrm{C}_{18} \mathrm{H}_{15} \mathrm{NO}_{3}$ : C, 73.71; $\mathrm{H}, 5.15 ; \mathrm{N}, 4.76 \%$.

spiro-4-(ethylenedioxy)-7-hydropyrrolo[3,2,1-de] phenanthridin-5-one (10 b)

mp. $161-162^{\circ} \mathrm{C}, \mathrm{IR} v_{\max } / \mathrm{cm}^{-1} 3060,2986,2903,1721$, 1633, 1489, 1440, 1367, 1312, 1283, 1194, 1148, 1002, 950, 756; Mass [ $\mathrm{m} / \mathrm{z}$ (abun.)] 279(32\%), 250(100), 220(3), 206(53), 190(4), 178(37), 151(36), 126(6), 103(28), 89(62), 76(71), 63(19); ${ }^{1} \mathrm{H}$ NMR $\left(300 \mathrm{MHz}, \mathrm{CDCl}_{3}\right) \delta$ 4.31-4.39 (m, $2 \mathrm{H}$, $\left.\mathrm{CH}_{2}\right), 4.57-4.62\left(\mathrm{~m}, 2 \mathrm{H}, \mathrm{CH}_{2}\right), 4.93\left(\mathrm{~s}, 2 \mathrm{H}, \mathrm{CH}_{2}\right), 7.04(\mathrm{t}, 1 \mathrm{H}$, $\mathrm{CH}, J$ 7.7), $7.13(\mathrm{~d}, 1 \mathrm{H}, \mathrm{CH}, J$ 7.2 Hz), 7.22-7.31 (m, 3H, CH), 7.65 (dd, $1 \mathrm{H}, \mathrm{CH}, J 0.6,8.0 \mathrm{~Hz}$ ), 7.73 (dd, 1H, CH, J 1.4, 7.3 $\mathrm{Hz}) ;{ }^{13} \mathrm{CNMR}$ PENDANT $\left(75 \mathrm{MHz}, \mathrm{CDCl}_{3}\right) \delta 42.3\left(\mathrm{CH}_{2}\right)$, 65.8 $\left(\mathrm{CH}_{2}\right), 103.1(\mathrm{C}), 117.4(\mathrm{C}), 122.4(\mathrm{CH}), 122.7(\mathrm{C}), 123.5$ $(\mathrm{CH}), 124.1(\mathrm{CH}), 124.3(\mathrm{CH}), 127.6(\mathrm{CH}), 127.7(\mathrm{C}), 128.0$ $(\mathrm{C}), 128.0(\mathrm{CH}), 128.5(\mathrm{CH}), 139.3(\mathrm{C}), 172.8(\mathrm{C}=\mathrm{O})$. Found: C, 73.03; H, 4.42; N, 5.08. Calc. for $\mathrm{C}_{17} \mathrm{H}_{13} \mathrm{NO}_{3}$ : C, 72.94; $\mathrm{H}, 4.64 ; \mathrm{N}, 5.01 \%$.

spiro-4-(ethylenedioxy)-2-nitro-7-hydropyrrolo [3,2,1-de]phenanthridin-5-one (10 c)

mp. $238-240^{\circ} \mathrm{C}$ (decomp.); IR $v_{\max } / \mathrm{cm}^{-1} 3083,2971$, 2909, 1739, 1613, 1527, 1493, 1339, 1252, 1175, 1031, 946, 781, 753, 729; Mass [ $\mathrm{m} / \mathrm{z}$ (abun.)] 324(24\%), 295(100), 251(39), 224(16), 205(26), 177(45), 150(32), 126(5), 103(16), 86(66), 75(79), 63(16); ${ }^{1} \mathrm{H} \mathrm{NMR}\left(200 \mathrm{MHz}, \mathrm{CDCl}_{3} /\right.$ DMSO-d $\left._{6}\right) \delta 4.16-4.22\left(\mathrm{~m}, 2 \mathrm{H}, \mathrm{CH}_{2}\right), 4.35-4.41(\mathrm{~m}, 2 \mathrm{H}$, $\left.\mathrm{CH}_{2}\right), 4.81\left(\mathrm{~s}, 2 \mathrm{H}, \mathrm{CH}_{2}\right), 7.02(\mathrm{~d}, 1 \mathrm{H}, \mathrm{CH}, J 5.3 \mathrm{~Hz}), 7.20(\mathrm{~m}$, $2 \mathrm{H}, \mathrm{CH}), 7.67$ (d, 1H, CH, J 5.4 Hz), 7.91 (s, 1H, CH), 8.42 (s, $1 \mathrm{H}, \mathrm{CH}) ;{ }^{13} \mathrm{C}$ NMR PENDANT $\left(50 \mathrm{MHz}, \mathrm{CDCl}_{3} / \mathrm{DMSO}-\right.$ $\left.\mathrm{d}_{6}\right) \delta 42.1\left(\mathrm{CH}_{2}\right), 65.9\left(\mathrm{CH}_{2}\right), 101.5(\mathrm{C}), 117.7(\mathrm{C}), 119.4$ $(\mathrm{CH}), 119.9(\mathrm{CH}), 122.7(\mathrm{CH}), 123.2(\mathrm{C}), 125.6(\mathrm{C}), 127.6$ $(\mathrm{CH}), 128.2(\mathrm{C}], 128.4(\mathrm{CH}), 129.8(\mathrm{CH}), 144.2(\mathrm{C}), 144.6$ (C), $172.9(\mathrm{C}=\mathrm{O}]^{*}$. Found: $\mathrm{C}, 63.13 ; \mathrm{H}, 3.45 ; \mathrm{N}, 8.81$. Calc. for $\mathrm{C}_{17} \mathrm{H}_{12} \mathrm{~N}_{2} \mathrm{O}_{5}$ : C, 62.94; H, 3.55; N, 8.86\%. *Signal observed in the normal ${ }^{1} \mathrm{H}$ decoupled ${ }^{13} \mathrm{C}$ spectrum recorded in $\mathrm{C}_{6} \mathrm{D}_{6} / \mathrm{CDCl}_{3}$.

spiro-4-(ethylenedioxy)-9,10-(methylenedioxy)-7-hydropyrrolo[3,2,1-de]phenanthridin-5-one (10 d)

mp. 226- $228^{\circ} \mathrm{C}$ (decomp.); IR $v_{\max } / \mathrm{cm}^{-1} 3047,2969$, 2900, 1716, 1638, 1508, 1475, 1369, 1238, 1188, 1153, 
1042, 939, 787, 744; Mass [m/z (abun.)] 323(42\%), 294(100), 264(25), 250(37), 223(27), 164(23), 138(14), 124(30), 110(14), 96(43), 82(39), 69(25), 63(14); ${ }^{1} \mathrm{H}$ NMR $\left(300 \mathrm{MHz}, \mathrm{CDCl}_{3}\right) \delta 4.31-4.39\left(\mathrm{~m}, 2 \mathrm{H}, \mathrm{CH}_{2}\right), 4.54-4.61$ (m, 2H, $\left.\mathrm{CH}_{2}\right), 4.88\left(\mathrm{~s} ; 2 \mathrm{H}, \mathrm{CH}_{2}\right), 6.00\left(\mathrm{~s}, 2 \mathrm{H}, \mathrm{CH}_{2}\right), 6.61(\mathrm{~s}$, $1 \mathrm{H}, \mathrm{CH}), 7.03(\mathrm{t}, 1 \mathrm{H}, \mathrm{CH}, J 7.5 \mathrm{~Hz}), 7.18(\mathrm{~s}, 1 \mathrm{H}, \mathrm{CH}), 7.19$ $(\mathrm{dd}, 1 \mathrm{H}, \mathrm{CH}, J \quad 0.7,7.5 \mathrm{~Hz}), 7.50(\mathrm{~d}, 1 \mathrm{H}, \mathrm{CH}, J 7.5 \mathrm{~Hz}) ;{ }^{13} \mathrm{C}$ NMR $\left(75 \mathrm{MHz}, \mathrm{CDCl}_{3}\right) \delta 42.9\left(\mathrm{CH}_{2}\right), 66.0\left(\mathrm{CH}_{2}\right), 101.8$ $\left(\mathrm{CH}_{2}\right), 102.9(\mathrm{CH}), 103.5(\mathrm{C}), 107.8(\mathrm{CH}), 117.8(\mathrm{C}), 122.3$ (C), $122.6(\mathrm{C}), 122.7(\mathrm{C}), 123.7(\mathrm{CH}), 123.8(2 \times \mathrm{CH}), 139.0$ (C), $148.1(\mathrm{C}), 148.3(\mathrm{C}), 173.1(\mathrm{C}=\mathrm{O})$.

spiro-4-(ethylenedioxy)-9,10-dimethoxy-7- hydropyrrolo[3,2,1-de]phenanthridin-5-one (10 e)

mp. $188-190^{\circ} \mathrm{C} ; \quad$ IR $v_{\max } / \mathrm{cm}^{-1} 3067,2966,2839$, 1719, 1635, 1467, 1358, 1217, 1185, 1149, 1048, 1002, 940, 854, 780, 737; Mass [m/z (abun.)] 339(42\%), 310(100), 266(21), 239(28), 153(8), 134(8), 126(5), 63(3); ${ }^{1} \mathrm{H} \mathrm{NMR}$ $\left(300 \mathrm{MHz}, \mathrm{CDCl}_{3}\right) \delta 3.89$ (s, $\left.3 \mathrm{H}, \mathrm{CH}_{3}\right), 3.95\left(\mathrm{~s}, 3 \mathrm{H}, \mathrm{CH}_{3}\right)$, 4.31-4.35 (m, 2H, $\left.\mathrm{CH}_{2}\right), 4.57-4-62\left(\mathrm{~m}, 2 \mathrm{H}, \mathrm{CH}_{2}\right), 4.88(\mathrm{~s}$, $\left.2 \mathrm{H}, \mathrm{CH}_{2}\right), 6.60(\mathrm{~s}, 1 \mathrm{H}, \mathrm{CH}), 7.03(\mathrm{t}, 1 \mathrm{H}, \mathrm{CH}, J 7.4 \mathrm{~Hz}), 7.16$ (s, $1 \mathrm{H}, \mathrm{CH}), 7.19(\mathrm{~d}, 1 \mathrm{H}, \mathrm{CH}, J 7.4 \mathrm{~Hz}), 7.53(\mathrm{~d}, 1 \mathrm{H}, \mathrm{CH}, J$ 7.4Hz); ${ }^{13} \mathrm{C} \mathrm{NMR}\left(75 \mathrm{MHz}, \mathrm{CDCl}_{3}\right) \delta 42.0\left(\mathrm{CH}_{2}\right), 55.9$ $\left(2 \times \mathrm{CH}_{3}\right), 65.7\left(\mathrm{CH}_{2}\right), 101.2(\mathrm{C}), 105.1(\mathrm{CH}), 109.9(\mathrm{CH})$, $117.5(\mathrm{C}), 120.5(\mathrm{C}), 121.0(\mathrm{CH}), 122.3(\mathrm{C}), 123.2(\mathrm{CH})$, $123.2(\mathrm{CH}), 123.4(\mathrm{CH}), 138.4(\mathrm{C}), 148.6(\mathrm{C}), 149.3(\mathrm{C})$, $172.8(\mathrm{C}=\mathrm{O})$; HRMS. Found: 339.1107. Calc. for $\mathrm{C}_{19} \mathrm{H}_{17} \mathrm{NO}_{5}: 339.1107$.

\section{Acknowledgements}

The authors thank CNPq, the PRONEX program and FAPERJ for financial support. SJG also thanks the Fundação Oswaldo Cruz, Farmanguinhos, Rio de Janeiro for support from the PAPES II program. The following are gratefully acknowledged: Prof. Marcos N. Eberlin (UNICAMP, São Paulo) for the HRMS and the Instituto de Química, USP, São Paulo, and CENPES, Rio de Janeiro, for the elemental analyses.

\section{References}

1. (a) Llabres, J. M.; Viladomat, F.; Bastida, J.; Codina, C.; Rubiralta, M. Phytochemistry 1986, 25, 2637; (b) Xiong, Y.; Moore, H. W. J. Org. Chem. 1996, 61, 9168.

2. see reference (1a); Bastida, J.; Viladomat, F.; Llabrés, J. M.; Codina, C.; Rubiralta, M. Planta Med. 1988, 54, 362.

3. (a) Ali, A. A.; El Sayed, H. M.; Abdallah, O. M.; Steglich, W. Phytochemistry 1986, 25, 2399; (b) Viladomat, F.; Codina, C.; Bastida, J.; Mathee, S.; Campbell, W. E. Phytochemistry 1995, 40, 961; (c) Queckenberg, O.
R.; Frahm, A. W.; MullerDoblies, D.; MullerDoblies, U. Phytochem. Analysis 1996, 7, 156.

4. (a) Ghosal, S.; Kumar, Y.; Singh, S. K.; Kumar, A. J. Chem. Res. (S) 1986, 112; (b) Abou-Donia, A. H.; De Giulio, A.; Evidente, A.; Gaber, M.; Habib, A. -A.; Lanzetta, R.; Seif El Din, A. A. Phytochemistry 1991, 30, 3445; (c) Abou-Donia, A. H.; Abib, A. A.; El Din, A. S.; Evidente, A.; Gaber, M.; Scopa, A. Phytochemistry 1992, 31, 2139; (d) Bastida, J.; Codina, C.; Porras, C. L.; Paiz, L. Planta Med 1996, 62, 74.

5. Hoshino, O. In The Alkaloids; Cordell, G. A., Ed.; Academic Press: San Diego, 1998; Vol. 51, p 323.

6. Consult reference (5); (a) Chattopadhyay, S.; Chattopadhyay, U.; Mathur, P. P.; Saini, K. S.; Ghosal, S. Planta Med 1983, 49, 252; (b) Zee-Cheng, R. K. Y.; Yan, S.-J.; Cheng, C. C. J. Med. Chem. 1978, 21, 199; (c) Ghosal, S.; Singh, S. K.; Kumar, Y.; Unnikrishnan, S.; Chattopadhyay, S. Planta Med 1988, 54, 114.

7. Consult reference (5); (a) For a recent review on the synthesis of biaryl compounds consult: Stanforth, S. P. Tetrahedron 1998, 54, 263; Coupling by nucleophilic aromatic substitution: (b) Hutchings, R. H.; Meyers, A. I. J. Org. Chem. 1996, 61, 1004; (c) Meyers, A. I.; Hutchings, R. H. Tetrahedron Lett. 1993, 34, 6185; (d) Reuter, D. C.; Flippin, L. A.; McIntosh, J.; Caroon, J. M.; Hammaker, J. Tetrahedron Lett. 1994, 35, 4899; Radical mediated couplings: (e) Rosa, A. M.; Prabhakar, S.; Lobo, A. M. Tetrahedron Lett. 1990, 31, 1881; (f) Lauk, U.; Dürst, D.; Fischer, W. Tetrahedron Lett. 1991, 32, 65; (g) Rosa, A. M.; Lobo, A. M.; Branco, P. S.; Prabhakar, S.; Pereira, A. M. D. L. Tetrahedron 1997, 53, 269; (h) Rosa, A. M.; Lobo, A. M.; Branco, P. S.; Prabhakar, S.; Sá-da-Costa, M. Tetrahedron 1997, 53, 299; (i) Tsuge, O.; Hatta, T.; Tsuchiyama, H. Chem. Lett. 1998, 155; Ullmann coupling: (j) Parnes, J. S.; Carter, D. S.; Kurz, L. J.; Flippin, L. A. J. Org. Chem. 1994, 59, 3497; Suzuki coupling: (k) Siddiqui, M. A.; Snieckus, V. Tetrahedron Lett. 1990, 31, 1523; (1) Geen, G. R.; Mann, I. S.; Mullane, M. V.; McKillop, A. Tetrahedron 1998, 54, 9875; Stille coupling: (m) Iwao, M.; Takehara, H.; Obata, S.; Watanabe, M. Heterocycles 1994, 38, 1717; Stoichiometric oxidative palladium coupling: (n) Black, D. St. C.; Keller, P. A.; Kumar, N. Tetrahedron 1993, 49, 151; Palladium mediated coupling by hydrogen halide elimination: (o) Ames, D. E.; Opalko, A. Tetrahedron 1984, 40, 1919; (p) Harayama, T.; Akiyama, T.; Kawano, K. Chem. Pharm. Bull. 1996, 44, 1634; (q) Harayama, T.; Akiyama, T.; Nakano, Y. Chem. Pharm. Bull. 1997, 45, 1723; (r) Harayama, T.; Shibaike, K. Heterocycles 1998, 49, 191. 
8. Heck, R. F. Org. React. 1982, 27, 345.

9. (a) de Meijere, A.; Meyer, F. A. Angew. Chem. Int. Ed. Engl. 1994, 33, 2379; (b) Cabri, W.; Candiani, I. Acc. Chem. Res. 1995, 28, 2; (c) Gibson, S. E.; Middleton, R. J. Contemp. Org. Synth. 1996, 3, 447; (d) Shibasaki, M.; Boden, C. D. J.; Kojima, A. Tetrahedron 1997, 53, 7371; (e) Crisp, G. T. Chem. Soc. Rev. 1998, 27, 427.

10. For unsuccessful attempts to cyclise 2-phenyl-7-bromo$N$-benzylindole and 2-phenyl- $N$-(o-bromoben-zyl)indole derivatives to the corresponding pyrrolophenanthridines see reference $(7 n)$; for successful cyclisations to benzophenanthridine derivatives but using larger quantities of palladium catalysts see references (7p-r); (a) Kraus, G. A.; Kim, H. Synth. Comm. 1993, 23, 55; (b) Sakamoto, T.; Yasuhara, A.; Kondo, Y.; Yamanaka, H. Heterocycles 1993, 36, 2597; (c) Desarbre, E.; Mérour, J. -Y. Heterocycles 1995, 41, 1987.

11. see reference (7n); (a) Tsuji, J. Palladium Reagents and Catalysts. Innovations in Organic Synthesis; John Wiley and Sons. Inc.: New York, 1995; (b) Heck, R. F. Palladium Reagents in Organic Synthesis; Academic Press, Inc.: London, 1985; (c) Black, D. St. C. Synlett 1993, 246; (d) Itahara, T. Synthesis 1979, 151.

12. Rigby, J. H.; Mateo, M. E. Tetrahedron 1996, 52, 10659.

13. (a) Torres, J. C.; Garden, S. J.; Pinto, A. C.; Silva, F. S. Q.; Boechat, N. Tetrahedron 1999, 55, 1881; (b) Pinto, A. C.; Silva, F. S. Q.; Silva, R. B. Tetrahedron Lett. 1994, 35, 8923; (c) Lopes, W. A.; Silva, G. A.; Sequeira, L. C.; Pereira, A. L.; Pinto, A. C. J. Braz. Chem. Soc.,
1993, 4, 34; (d) Wierenga, W.; Griffin, J.; Warpehoski, M. A. Tetrahedron Lett. 1983, 2437; (e) Sirowej, H.; Khan, S. A.; Pleininger, H. Synthesis 1972, 84.

14. (a) Gribble, G. W. Chem. Soc. Rev. 1998, 395; (b) Gribble, G. W.; Hoffman, J. H. Synthesis 1977, 859; (c) Gribble, G. W.; Lord, P. D.; Skotnicki, J.; Dietz, S. E.; Eaton, J. T.; Johnson, J. L. J. Am. Chem. Soc. 1974, 96, 7812.

15. Garden, S. J.; Torres, J. C.; da Silva, L. E.; Pinto, A. C. Synth. Commun. 1998, 28, 1679.

16. A typical experiment involved refluxing an $\mathrm{CH}_{3} \mathrm{CN}$ solution $\left(0.05 \mathrm{~mol} \mathrm{~L}^{-1}\right)$ of the substrate in the presence of 10 mole $\% \mathrm{Pd}(\mathrm{OAc})_{2}, 20$ mole $\% \mathrm{Ph}_{3} \mathrm{P}$ and 2 equivalents of $\mathrm{Et}_{3} \mathrm{~N}$.

17. Martinez, F.; Naarmann, H. Synthet. Metal 1990,39, 195. 18. (a) Lathourakis, G. E.; Litinas, K. E. J. Chem. Soc. Perkin Trans. 1 1996, 491; (b) Boulos, L. S.; El-Kateb, A. A. Chem. Ind. (London) 1983, 864.

19. (a) Crestini, C.; Saladino, R. Synth. Commun. 1994, 24, 2835; (b) Soriano, D. S. J. Chem. Educ. 1993, 70, 332.

20. Prepared by reaction of the known $N$-benzylisatin (see reference 15) with ethylene glycol in the presence of $\mathrm{H}_{2} \mathrm{SO}_{4}$.

21. (a) Jeffery, T. Tetrahedron 1996, 52, 10113; (b) Jeffery, T. J. Chem. Soc. Chem. Commun. 1984, 1287.

22. For references to phosphine free Heck reactions see reference (21a) and references cited in Reetz, M. T.; Westermann, E.; Lohmer, R.; Lohmer, G. Tetrahedron Lett. 1998, 39, 8449.

Received: January 21, 2000 Published on the web: August 31, 2000 